\title{
A Tool Path Generation Method for Sculptured Surface Based on the Vector Field of Maximum Strip Width
}

\author{
Cheng $\mathrm{Su}^{1}$, Xin Jiang ${ }^{1, *}$, Guanying Huo ${ }^{1}$, Danlei $\mathrm{Ye}^{1}$, Zehong $\mathrm{Lu}^{2}$, Bolun Wang ${ }^{1}$ and Zhiming Zheng ${ }^{1}$ \\ ${ }^{1}$ LMIB and School of Mathematics and Systems Science, Beihang University, Beijing, China \\ ${ }^{2}$ School of Mathematical Science, Peking University, Beijing, China \\ ${ }^{3}$ Big Data Brain Computing Center, Beihang University, Beijing, China \\ *Corresponding author
}

\begin{abstract}
For three-axis sculptured surface machining, a tool path generation method based on the feed directions of maximum machining strip width is proposed. We use a B-Spline stream function to reconstruct the vector field of feed directions, and give the initial tool path by one of the iso-lines of the stream function. The integrated tool paths are generated using traditional iso-scallop method based on the previous obtained initial path. Numerical results show that the tool paths match the optimal vector field in the least square sense. Further a total shorter tool path length can be obtained.
\end{abstract}

Keywords-tool path; streamline function; vector fied

\section{INTRODUCTION}

Sculptured surfaces have been widely applicated in designing complex parts in modern industry, such as automotive, aerospace and navigation, while the process of sculptured surfaces machining is time-consuming. With the development of geometric accuracy and reliability of advanced parts with complex surfaces, the NC machining of this type of parts has been focused on the assurance of machining accuracy and efficiency. Thus the toolpath planning becomes one of the key steps in NC machining.

Conventional methods include iso-parametric [1-3] and isoscallop methods $[4,5]$. The iso-parametric method is the earliest means which fixes one parameter and varies the other to generate tool paths. The pros and cons of this method are obvious. The convenience in mathematical expression and computing is along with quite a lot of redundant machining due to the inadequate use of the maximum side-step. As a solution, iso-scallop method was proposed. It ensures that scallop height between the adjacent paths remains the special limit which is equivalent to the maximum side-step. However, the overall length is determined by the initial tool path and the geometric property of the surface. The selection of the initial tool path is vital in iso-scallop method.

The evaluation about the tool path mainly refers to the quality and efficiency. First, the tolerance, which is to measure the quality of the surface, should not be violated. Then how to reduce the machining time as much as possible is taken into consideration. An intuitive thought is that the shorter total length of tool paths leads to the more efficient machining.
When the cutter and machining tolerance is determined, the feed direction is the only variable for the machining strip width in three-axis machining using ball-end mills. According to the various curvature of the surface, different feed direction results in the different scallop height, which has positive correlation with the side-step in the maximum tolerance. Larger side-step between the adjacent paths leads to the less number of tool paths, which contributes to a short overall machining tool path length.

A series of methods have been proposed to obtain the optimal feed directions. A metric designed by the abstract Riemannian manifold is used to describe the curvature tensors of the cutter surface and the part surface [6]. Recently a method was proposed to obtain feed direction with the maximum machining strip width derived by rank-two tensor field [7]. At the same time the tensor field method was used to divide the surface into sub-surface regions by the degenerate points [8].

In this paper, the dynamic process of machining is simulated as the movement of incompressible fluids. The vector field in the parametric domain can be generated by the feed direction of maximum machining strip width. Then the planar continuity equation is used to reconstruct the vector field. A B-Spline form function is constructed to generate tool paths. The zero-value line of the function is selected as the initial tool path, and the further adjacent tool paths are generated using iso-scallop method.

\section{OPTIMAL FEED DIRECTION}

The machining strip width is limited by the scallop height tolerance. Lee and Ji proposed the notion and obtained the optimal cutter feed direction for the five-axis machining [9]. The potential field method treat the machining strip width as scalar at every cutter contact (CC) point [10]. By analyzing the geometry between the cutter surface and part surface, the vector field which is related to the maximum machining strip width can be generated.

The parameterizations of the part surface and cutter surface are defined as $S_{\text {part }}=P(u)$ and $S_{\text {cutter }}=C(\alpha)$ respectively, where $u=\left[u^{1}, u^{2}\right]^{T}$ and $\alpha=\left[\begin{array}{ll}\alpha^{1}, & \alpha^{2}\end{array}\right]^{T}$ are 
the parameters of correlative surfaces. A local co-ordinate system is described by $\left\{X_{p}, Y_{p}, Z_{p}\right\}$ at the CC points $P\left(u_{0}\right)$. The CC point $P\left(u_{0}\right)$ of the part surface also is the cutter surface point $C\left(\alpha_{0}\right)\left(P\left(u_{0}\right)=C\left(\alpha_{0}\right)\right) . X_{p}$ is the feed direction, $Z_{p}$ is the normal vector of the part surface at $P\left(u_{0}\right)$, and the $Y_{p}=X_{p} \times Z_{p}$. The scallop height $h_{s}$ should not be larger than the given tolerance $\varepsilon_{h}$. Regarding $h_{s}$ as a variable value of parameter $u$, the limitation $h_{s}(u) \leq \varepsilon_{h}$ is given.

When using the ball-end mills, the tangent planes of the part surface and the cutter surface are the same at the CC point due to the same normal vector. Let $N(u)$ represent the unit vector at $P(u)$ which is parallel to the normal vector $Z_{p}$ at the CC point $P\left(u_{0}\right)$. Let $h(u)$ represent the height at in $N(u)$ direction from the point $P(u)$ of the part surface to the nearest cutter surface point. The height $h(u)$ can be considered to the scallop height $h_{s}(u)$ approximately.

The $h(u)$ could be attained by the equation

$$
P(u)+h(u) \cdot N\left(u_{0}\right)=C^{u_{0}}(\alpha),
$$

where $C^{u_{0}}(\alpha)$ is the point of the sphere cutter surface which makes $\overline{P(u) C^{u_{0}}(\alpha)}$ parallel to $N(u)$.

Not introducing the Gauss Derivation Formula into (1), the $h(u)$ has another geometric description.

$$
\begin{aligned}
& h(u)=\overline{P\left(u_{0}\right) C^{u_{0}}(\alpha)} \cdot \overline{N\left(u_{0}\right)} \\
& -\overline{P\left(u_{0}\right) P(u)} \cdot \overline{N\left(u_{0}\right)} .
\end{aligned}
$$

$\overline{P\left(u_{0}\right) C^{u_{0}}(\alpha)} \cdot \overline{N\left(u_{0}\right)}$ and $\overline{P\left(u_{0}\right) P(u)} \cdot \overline{N\left(u_{0}\right)}$ are the second fundamental form of the cutter surface and the part surface respectively.

As we all know, the second fundamental form characterizes the degree of curvature of a surface along a specific direction at the point. The second fundamental form of the cutter surface and the part surface could be rewritten as

$$
I I_{\text {cutter }}(\alpha)=I I_{\text {cutter }}\left(\alpha^{1}, \alpha^{2}\right)=\overline{P\left(u_{0}\right) C^{u_{0}}(\alpha)} \cdot \overline{N\left(u_{0}\right)}
$$

and

$$
I I_{\text {part }}(u)=I I_{\text {part }}\left(u^{1}, u^{2}\right)=\overline{P\left(u_{0}\right) P(u)} \cdot \overline{N\left(u_{0}\right)} .
$$

The curvature $K_{\text {cutter }}$ of the cutter surface remains the same. By introducing the equation $K_{\text {cutter }}=I I_{\text {cutter }} / I_{\text {cutter }}$, where $I_{\text {cutter }}$ is the first fundamental form of the cutter surface, $h$ could be represents as

$$
\begin{aligned}
& h(u)=I I_{\text {cutter }}-I I_{\text {part }} \\
& =K_{\text {cutter }} \cdot I_{\text {cutter }}-I I_{\text {part }}
\end{aligned}
$$

With the Taylor expansion, $I_{\text {cutter }}$ could be replaced with $I_{\text {part }}$ approximately. Then further expression of $h$ could be represented as

$$
h(u) \approx\left[u-u_{0}\right]^{T} H\left(u_{0}\right)\left[u-u_{0}\right],
$$

where

$$
\begin{aligned}
& H(u)=\frac{1}{2}\left(K_{\text {cutter }} \cdot \widetilde{I_{\text {part }}}(u)-\widetilde{I I_{\text {part }}}(u)\right) \\
& =\frac{1}{2}\left[\begin{array}{ll}
K_{\text {cutter }} P_{u^{1}} P_{u^{1}}-N\left(u_{0}\right) P_{u^{1} u^{1}} & K_{\text {cutter }} P_{u^{1}} P_{u^{2}}-N\left(u_{0}\right) P_{u^{1} u^{2}} \\
K_{\text {cutter }} P_{u^{2}} P_{u^{1}}-N\left(u_{0}\right) P_{u^{2} u^{1}} & K_{\text {cutter }} P_{u^{2}} P_{u^{2}}-N\left(u_{0}\right) P_{u^{2} u^{2}}
\end{array}\right] .
\end{aligned}
$$

$\widetilde{I_{\text {part }}}(u)$ and $I I_{\text {part }}(u)$ are the first and second fundamental form coefficient matrixes of the part surface.

Then the tensor property is introduced to obtain the direction with the maximum machining strip width. The inverse matrix of $\widetilde{I_{\text {part }}}(u)$ is used in order to make the matrix of approximate height satisfy the tensor operation rules.

$$
T=H \cdot I_{p a r t}^{-1}
$$

The eigenvector which is related to the largest absolute value of the eigenvalue represents the feed direction with the largest $h$. The vector field of the maximum machining strip width at any point on the surface could be obtained. The feed directions of the maximum machining strip width at some sample points are shown in Figure 1. And the vector field of the parameter domain are shown in Figure 2. 


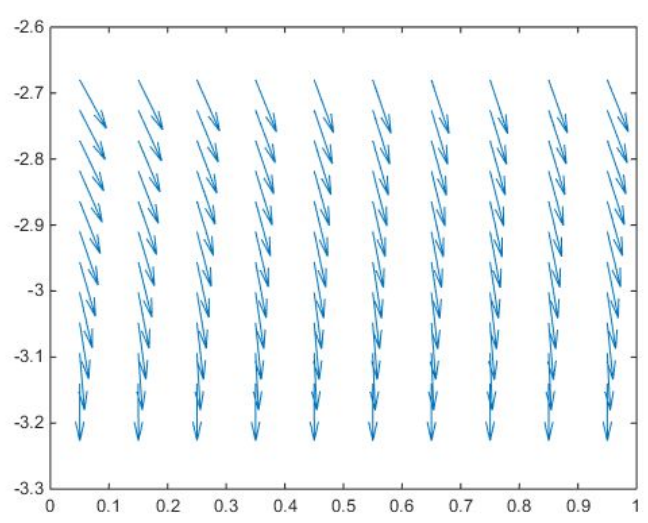

FIGURE I. VECTOR FIELD OF PARAMETER DOMAIN

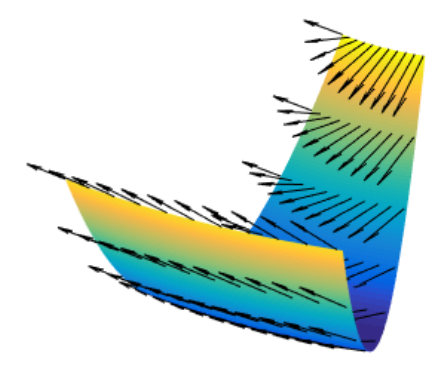

FIGURE II. FEED DIRECTIONS OF THE MAXIMUM MACHINING STRIP WIDTH

\section{CONSTRUCtion OF THE TOOL PATH EQUATION}

The vector field supplies the information about point positions and the corresponding directions. It guides the movement of the ball-end mills. Some methods were proposed to reconstruct the vector field $[9,13,14]$. The smooth tool path whose tangent vectors are parallel to the directions at the same points is considered to a good path. Meanwhile, the intersection of paths and the cusps need to be avoided. Sun et al. constructed a stream function by the gradient of the vectors [9]. Inspired by the method, we regard the movement of tool as the particle motion in the fluid. In this paper, a differential equation of incompressible fluid is used to fit the vector field.

\section{A. Fomulation of the Streamlines}

Streamlines are one type of field lines in a fluid flow, which show the motion of the flow field at the same time. There are some similarities between the desired movement of the tool and the streamlines of fluid flow.

- Streamlines are tangent to the velocity vector of the flow instantaneously in the whole field.

- There is no intersection between different streamlines due to the inexistence of two different velocities at the same point.

- Streamlines are a family of smooth curves in which the cusps do not exist.
Comparing with the vector field that has been generated, let $V=\left(\tau^{\|}, \tau^{\perp}\right)$ be the velocity field of a steady incompressible, irrotational flow in two dimensions, where the parameters $\tau^{\|}$ and $\tau^{\perp}$ are the horizontal and vertical components.

Due to the incompressibility of the flow the condition could be represented as

$$
\nabla V=\frac{\partial \tau^{\|}}{\partial u}+\frac{\partial \tau^{\perp}}{\partial v}=0
$$

The condition of the irrotationality is that

$$
\nabla \times V=\frac{\partial \tau^{\perp}}{\partial u}-\frac{\partial \tau^{\|}}{\partial v}=0
$$

The stream function $\Psi$ is defined by the differential

$$
d \Psi=\tau^{\perp} d u-\tau^{\|} d v=0
$$

It is because that the incompressible condition is integrable. Note that it is constant along the streamlines, the value of the stream function could be used to define a tool path.

The first derivatives of the stream function $\Psi$ are given by

$$
\frac{\partial \psi}{\partial u}=\tau^{\perp}, \frac{\partial \psi}{\partial v}=-\tau^{\|}
$$

\section{B. Reonstruction of the Vector Field}

The vector field supplies some discrete points and directions on the parameter domain. And the stream function could be represented by several forms. B-Spline form is selected due to its flexibility for locally fitting.

The vector field includes the sample points' positions $\left\{p_{s}=\left(u^{s}, v^{s}\right) \mid s=1,2, \ldots, m\right\}$ and the corresponding vectors $\left\{\tau_{s}=\left(\tau_{s}{ }^{u}, \tau_{s}{ }^{v}\right) \mid s=1,2, \ldots, m\right\}$.

A stream function with B-Spline form is defined by

$$
\Psi(u, v)=\sum_{i=0}^{m} \sum_{j=0}^{n} d_{i, j} N_{i, k}(u) N_{j, l}(v), u, v \in[0,1]
$$

The gradient of the function at a sample point $p_{i}$ is that 


$$
\begin{aligned}
& \nabla \Psi\left(p_{i}\right)=\left[\begin{array}{ll}
\frac{\partial \Psi\left(u^{i}, v^{i}\right)}{\partial u} & \left.\frac{\partial \Psi\left(u^{i}, v^{i}\right)}{\partial v}\right]^{T} \\
= & \left.\tau_{i}^{v},-\tau_{i}^{u}\right]^{T}, i=1, \ldots, m
\end{array}\right.
\end{aligned}
$$

The knots of parameters $u$ and $v$ could be chosen by the groups of sample points. As the solution of an overdetermined system of equations, the control points of the stream function would be gotten in the least squares sense.

\section{ToOl Paths Generation BASEd on the StREAM FUNCTION}

The Streamlines has some advantages when selected as the tool paths, such as the smoothness and the property of intersection inexistence. In addition, the value of the stream function must be constant along a streamline since streamlines are tangent to the flow velocity vector.

\section{A. Toolpath Generation Using Iso-Lines Method}

Instead of parameters $u$ and $v$, the only parameter of toolpath determination is the value of stream function. Sun et al. presented a method that all tool paths are streamlines [11]. It is a method that is similar to iso-parametric method actually.

In this method, two steps would iterate when generating tool paths. The parameter pairs of the selected value of streamline $\left\{\left(u^{C_{i}}, v^{C_{i}}\right) \mid \Psi\left(u^{C_{i}}, v^{C_{i}}\right)=C_{i}\right\}$ are computed, and offsetting for the corresponding points occurs later.

Through partitioning the parameter domain, the values of stream function at the grid are gotten. Then approximate values of the whole field could be obtained by linear interpolation.

Let $L_{i}: \Psi(u, v)=C_{i}$ be a selected streamline, on which is a point $p_{k}=S_{\text {part }}\left(u^{k}, v^{k}\right)$. And $p_{k}^{*}$ is the offset point of $p_{k}$. The feed direction is perpendicular to the side-step on the part surface

$$
\left(p_{k}^{*}-p_{k}\right) \cdot L_{i}^{\prime}\left(u^{k}, v^{k}\right)=0
$$

where the $L_{i}^{\prime}\left(u^{k}, v^{k}\right)$ is the tangent vector of streamline $L_{i}$ at $\left(u^{k}, v^{k}\right)$.

According to the scallop tolerance, the length between $p_{k}$ and $p_{k}^{*}$ should be limited as follow

$$
\left\|p_{k}^{*}-p_{k}\right\| \leq \varepsilon_{\text {length }}\left(u^{k}, v^{k}\right)
$$

where $\varepsilon_{\text {length }}$ is derived from the local geometry property and the scallop tolerance [15].

By applying the Taylor expansion of the part surface, $p_{k}^{*}$ could be associated with $p_{k}$ as follow

$$
p_{k}^{*}=p_{k}+P_{k}^{u} \Delta u^{k}+P_{k}^{v} \Delta v^{k}
$$

where $P_{k}^{u}$ and $P_{k}^{v}$ are the partial derivatives at $\left(u^{k}, v^{k}\right)$.

After computing $\Delta u^{k}$ and $\Delta v^{k}$, the offset point is gotten as follow

$$
p_{k}^{*}=S_{p a r t}\left(u^{k}+\Delta u^{k}, v^{k}+\Delta v^{k}\right)
$$

For all offset points, the values of stream function are obtained by

$$
C_{i+1}^{k}=\Psi\left(u^{k}+\Delta u^{k}, v^{k}+\Delta v^{k}\right)
$$

The value of next streamline is the stream function value at the offset point where the value of $\left|C_{i+1}^{k}-C_{i}\right|$ is minimum.

\section{B. Toolpath Generation to Shorten the Toolpath Length}

Though the above method ensures that the all tool paths are streamlines, the redundant machining reduces the machining efficiency. Searching for the shorter overall toolpath length, the streamline is chosen as the initial tool path, from which the more adjacent tool paths are generated by iso-scallop method.

Using the boundary as the initial tool path, we find that the tangent vector of the tool paths, which are generated from the initial tool path, do not match the vector field at most sample points. And the same situation was discussed [8].

The middle value of stream function is selected as value of the initial streamline (In general, 0 can be chosen as the initial value). Smaller deformation of the tool paths from the initial streamline occurs.

\section{EXAMPLES AND TEST RESUlts}

The test surface is described by

$$
\left\{\begin{array}{c}
x=(60 u+20) \cos (v) \\
y=(60 u+20) \sin (v) \\
z=15 u^{3}+15 u^{2}-30 u+20
\end{array}\right.
$$

$$
0.05 \leq u \leq 0.95,-\pi \leq v \leq-0.85 \pi
$$


A ball-end mill, of which the diameter is $5 \mathrm{~mm}$, is utilized here. The scallop height is limited within $0.1 \mathrm{~mm}$. By choosing the 0 -value of streamlines as the initial tool path, the rest tool paths are generated as shown in Figure 3. For comparison, the iso-parametric method, iso-scallop method and the stream function method are tested, as shown in Figure 4-6 respectively. The overall tool path lengths of the above four methods are shown in Table 1.

TABLE I. COMPARISON IN TOOL PATHS LENGTH

\begin{tabular}{|l|l|c|}
\hline \multicolumn{1}{|c|}{ Methods } & $\begin{array}{c}\text { Total tool path } \\
\text { length }(\mathbf{m m})\end{array}$ & $\begin{array}{c}\text { Reduction in } \\
\text { tool path length }\end{array}$ \\
\hline Iso-parametric method & 1077.80 & $40.66 \%$ \\
\hline Iso-scallop method & 678.89 & $5.79 \%$ \\
\hline Stream function method & 774.63 & $17.44 \%$ \\
\hline The proposed method & 639.55 & - \\
\hline
\end{tabular}

Though tool paths generated by using the stream function method matches the directions of the maximum machining strip width best, the redundant machining lead to the longer tool path length. Not only does the tool paths generated by the proposed method match the directions in most of surface region, but the overall tool path length is the shortest in the four methods.

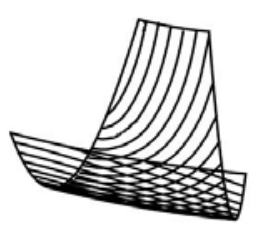

FIGURE III. THE PROPOSED METHOD TOOL PATHS

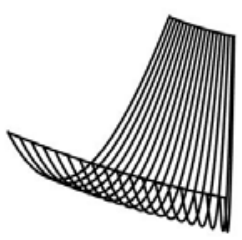

FIGURE IV. ISO-PARAMETER METHOD TOOL PATHS

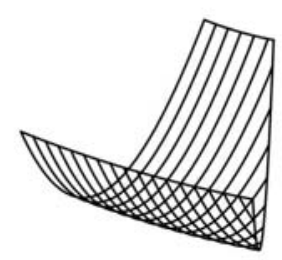

FIGURE V. ISO-SCALLOP METHOD TOOL PATHS

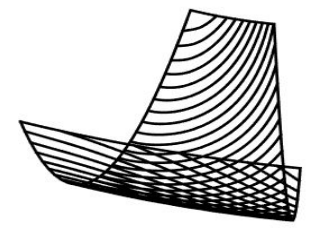

FIGURE VI. STREAM FUNCTION METHOD TOOL PATHS

\section{CONCLUSION}

This paper proposes a new tool path generation method for three-axis ball end mills. Considering the similarities between the streamlines and the desired tool path, we construct a stream function to give the tool path. In addition, aiming at reducing the length of overall tool paths, we select the middle position streamline (0-value) as the initial tool path. This method is tested and compared with iso-parametric method, iso-scallop method and the stream function method. It shows that the proposed method could achieve a better result in total tool paths length. As a further application, this method could be used for the vector field generated by other different criterions.

\section{ACKNOWLEDGMENT}

This work is partially supported by the Chinese National Science Foundation under Grant No.11290141, No.11571028.

\section{REFERENCES}

[1] Loney G C, Ozsoy T M. NC machining of free form surfaces[J]. Computer-Aided Design, 1987, 19(2):85-90.

[2] Elber G, Cohen E. Toolpath generation for freeform surface models[C]// on the Second ACM Symposium on Solid Modeling and Applications. DBLP, 1993:419-428.

[3] He W, Lei M, Bin H. Iso-parametric CNC tool path optimization based on adaptive grid generation[J]. The International Journal of Advanced Manufacturing Technology, 2009, 41(5):538-548.

[4] Suresh K, Yang D C H. Constant Scallop-height Machining of Freeform Surfaces[J]. Journal of Engineering for Industry, 1994, 116(2):253259.

[5] Ding S, Mannan M A, Poo A N, et al. Adaptive iso-planar tool path generation for machining of free-form surfaces[J]. Computer-Aided Design, 2003, 35(2):141-153.

[6] Kim T. Constant cusp height tool paths as geodesic parallels on an abstract Riemannian manifold[J]. Computer-Aided Design, 2007, 39(6):477-489.

[7] Liu X, Li Y, Ma S, et al. A tool path generation method for freeform surface machining by introducing the tensor property of machining strip width[J]. Computer-Aided Design, 2015, 66:1-13.

[8] Kumazawa G H, Feng H Y, Fard M J B. Preferred feed direction field: A new tool path generation method for efficient sculptured surface machining 2 [J]. Computer-Aided Design, 2015, 67-68:1-12.

[9] Y.S. Lee, H. Ji. Surface interrogation and machining strip evaluation for 5-axis CNC die and mold machining[J]. International Journal of Production Research, 1997, 35(1):225-252.

[10] Chiou C J, Lee Y S. A machining potential field approach to tool path generation for multi-axis sculptured surface machining[J]. 2002, 34(5):357-371.

[11] Sun Y. VECTOR ANALYSIS BASED TOOLPATH GENERATION FOR PRECISION SURFACE MACHINING[J]. Chinese Journal of Mechanical Engineering, 2005, 41(3):160-160. 
[12] Fard M J B, Feng H Y. Effect of tool tilt angle on machining strip width in five-axis flat-end milling of free-form surfaces[J]. The International Journal of Advanced Manufacturing Technology, 2009, 44(3):211-222.

[13] Mussa-Ivaldi F A. From basis functions to basis fields: vector field approximation from sparse data[J]. Biological Cybernetics, 1992, 67(6):479-489

[14] Kim T, Sarma S E. Toolpath generation along directions of maximum kinematic performance; a first cut at machine-optimal paths[J]. Computer-Aided Design, 2002, 34(6).

[15] Suresh K, Yang D C H. Constant Scallop-height Machining of Freeform Surfaces[J]. Journal of Engineering for Industry, 1994, 116. 